\title{
Review Article \\ Ensuring the First Breath: A Growing Accountability of Midwifery in Bangladesh
}

\author{
Muhammad Rehan Masoom \\ School of Business \& Economics, United International University, Dhaka, Bangladesh \\ Correspondence should be addressed to Muhammad Rehan Masoom; rehan_1611@yahoo.com
}

Received 27 September 2016; Revised 12 December 2016; Accepted 15 February 2017; Published 12 March 2017

Academic Editor: Sally Guttmacher

Copyright ( 2017 Muhammad Rehan Masoom. This is an open access article distributed under the Creative Commons Attribution License, which permits unrestricted use, distribution, and reproduction in any medium, provided the original work is properly cited.

\begin{abstract}
Successful women empowerment relies on providing quality infrastructure facilities to avail maternal healthcare on the national level. In this regard, ensuring women's access to quality midwifery services is an important consideration. The major intervention for safe maternity is to be enhanced to enable the presence of the skilled midwifery to ensure the quality emergency obstetric care. Therefore, the scope of practice of a midwife is very critical in the supervision of the orderly physiological processes of pregnancy, labor, birth, and the postpartum phase. The midwife as an autonomous practitioner is expected to be competent and accountable for her practice. In Bangladesh, the number of women having the baby at home by unskilled personnel is quite high. Therefore, the government strives to educate midwives and commits to reducing maternal and newborn mortality and morbidity. This study explores the contemporary situation of maternity health to provide a critical understanding of the growing importance of the role of midwifery in Bangladesh. With this, the paper examines the way maternity services in Bangladesh transformed from a social to a medical model over the twentieth century and infers how the social agenda was part of this process.
\end{abstract}

\section{Introduction}

More than one-hundred and thirty million humans take their first breath in this world each year. This is the moment of excitement for the mothers, and a time of joy for the people all around. However, for thousands of others, it often becomes the time of grief and sorrow; each year nearly three million children die in their first month of life [1]. World Health Organization (WHO) reports that nearly 830 females die from preventable conditions associated with pregnancy and childbirth every day [2]. Katie Taylor, the deputy child and maternal survival coordinator at USAID, mentioned that "any women need to able to access the care and services she needed to ensure that her next pregnancy had a happier ending: a safe pregnancy, a successful delivery and a healthy baby [sic]" [1]. One of the key healthcare components of maternal healthcare improvement is ensuring the presence of a certified birth attendant, often known as the midwife, at the time of delivery. Nonetheless, the reality of providing these needs is often socially complicated, economically difficult, and culturally challenging. Bangladesh is among the few nations that have made notable progress in the development of maternal health; however lack of trained midwife at the time of birth remains particularly a great concern. Bangladesh Demographic and Health Survey (2014) indicates that the trained midwife aided deliveries increased from 16 percent in 2004 to 42 percent in 2014; nonetheless, seeking midwifery guidance is alarmingly low among the less educated people living in remote areas.

There are above five thousand pregnancy-related deaths of women and seventy-six thousand neonatal deaths, and more than eighty stillbirths are reported every year in Bangladesh [3]. Bangladesh was positioned in the top ten nations that have the highest stillbirth rates [4]. Wordbank reports that the current maternal mortality rate (MMR) is 170 per 100,000 live births, which were 194 per hundred thousand in 2011. Though the MMR has been maintained considerably low for the last few years, nonetheless, the country should make efforts to reduce it to 70 per 100,000 live births by 2030 to fulfill the Sustainable Development Goals (SDG) commitment. According to Bangladesh Demographic and Health Survey (2014), only 42.1 percent of births had taken place with the presence of a skill birth attendant. About half of perinatal deaths per year happen as a direct outcome of 
poorly handled deliveries [5]. In addition, suboptimal care has been categorized in about 77 percent of perinatal deaths in developing nations like Bangladesh [2]. About one-fourth of the women at the time of delivery were underweight and around fifteen percent of them often have the diminutive stature that raises the risk of complicated childbirth and lowbirth-weight newborns. Fifty percent of all women suffer as a result of anemia. In addition, stunning strikes near about forty percent of children before they reach five years of age [3]. WHO reports, in Bangladesh, about 63.9 percent of women in their pregnancy visit antenatal care (ANC) at least once, against a minimum standard of four-time visits [6]. Near about 60 percent of the deliveries do not happen in health facilities [3]. Delivery at any healthcare facility is about 15 percent at its lowest, and delivery with the aid of a trained birth attendant is merely 18 percent [7]. Less qualified (or untrained and unprofessional) caregivers attend over 90 percent of these deliveries [8]. In a situation as such, an aggressive initiative needs to be taken to meet the MGD, and midwifery appears to be the best possible alternative.

Midwifery is globally recognized honorable professions as its benefits to bring a new life to the world in a dignified way for both the mother and the baby. It is considered one of the oldest callings in the world since it deals with reproduction. The State of the World's Midwifery Report (2014) has recognized women having access to midwifery-led care as crucial to attaining target objectives in all reproductive health sectors [9]. Global evidence suggests that any skilled midwife can contribute to 87 percent of the expected fundamental care for women and newborns and can handle almost fifty percent of the deliveries in a union [7]. Unions are the smallest rural administrative and local government unit. Midwives can be required to provide services like postpartum family planning. This indicates the need for midwife-led maternal and newborn healthcare scheme. Although "midwifery" is a highly respectable career and midwives need to be trained well to meet the quality service to uphold that respect, nonetheless ensuring the first breath of a human is not only a matter of health service availability, there are some socialeconomic facets involved into the issue as well. This study explores the contemporary situation of maternity health to provide a critical understanding of the growing importance of the role of midwifery in Bangladesh. With this, the paper examines the way maternity services in Bangladesh changed from a social to a medical model over the twentieth century and infers how the social agenda was part of this process. The study sheds lights on the present situation of midwifery education in Bangladesh and intends to outline how to make people aware of the benefits of having trained birth attendants around. In doing so, the paper addresses two fundamental issues: one is the training and educational aspects of midwifery, and the other is the socioeconomic issues related to the childbirth.

\section{Discussion}

The definition, as well as the role of the birth-attendants, differs from time to time in the country. There were Lady Family Planning Health Visitors, Lady Health Visitors,
Family Welfare Assistants (FWAs), Health Assistants (HAs), Traditional Birth Attendants (TBAs), Skilled Birth Attendants (SBAs), nurse, nurse-midwives, and midwives along with general practitioners and obstetricians-gynecologists. We have to outline the traits of midwifery in very straight terms to assess the role of midwife to avoid the potential scopes for conflict of interest among the medical professionals available in the country. With this regard, a history of the profession as such needs to be outlined.

The colonial legacy played a crucial role in shaping the healthcare system in Bangladesh. In 1973-74, there were near about eight hundred and fifty-six registered midwives, out of which the overwhelming majority was the nurse-midwives, along with few Lady Family Planning Health Visitors and Lady Health Visitors [10]. Although the midwives, nurses, and health visitors council had regulations concerning the midwifery services, nonetheless, no registration was needed for midwifery practice. In 1974, there were 15 midwifery training schools that had the capacity of preparing 240 midwives. Generally, the non-nurse-midwives need to have an eighteen-month duration of training, along with eight years of schooling, was required. On the other hand, oneyear training was necessary for the nurse-midwives. A 1975 national policy directed to control the population led the "family planning" wing of the Ministry of Health and Family Welfare (MoHFW) to the recruitment of thousands of fulltime female FWAs. Since then, the integration of maternal and child healthcare for family planning has been specified in the national health policies. Basically, the FWAs were referred to provide antenatal care. However, preservice training of FWAs and female HAs does not incorporate midwifery skills [11]. Afterward, in the 1970s, the distinct value of midwives dissolved, and the nurse-midwives replaced it. In 1987, the International Centre for Diarrhoeal Disease Research, Bangladesh (ICDDR, B), started a communitybased maternity care and recruited two government-trained nurse-midwives to conduct home deliveries, anticipate complexities, and lead them to the district hospital [12]. In the same year, a facility-based approach gained momentum due to the inception of the Safe Motherhood Initiative [13]. In 1996, ICDDR, B shifted their strategy from home-based to facility-based delivery; the community-based midwives were withdrawn. From 1993 to 1997, the WHO, United Nations Fund for Population Activities (UNFPA), Department for International Development (DFID), United Nations International Children's Emergency Fund (UNICEF), and other development partners supported government projects to establish Emergency Obstetric Care (EmOC) in the country.

Government health facilities are accessible down to union level; nonetheless, due to the existing social practices and cultural beliefs, an overwhelming majority of the deliveries occur at home. TBAs assist in three-quarters of births, of which 12 percent are assisted by trained and other 64 percent by untrained TBAs [14]. Medically trained cadres, such as doctors, nurses, family welfare visitors, midwives, and other medical assistants support only 13 percent of the deliveries. It is noteworthy that doctors or trained medical professionals assisted deliveries are substantially higher in the case of lower-order births, wealthy households, and 
mothers with secondary education [15]. Further, the severe deficit of nurses in Bangladesh limits existing registered nurse-midwives from being entirely utilized for midwifery activities. Primarily, serving as hospital staff nurses fulfilling vacancies and continually rotating throughout the hospital, registered nurse-midwives often lack the specialized inservice training. Registered nurse-midwives are under the direction of the Directorate of Nursing Service (DNS). Their chief working station is at tertiary level hospitals and sometimes the medical college hospitals or district hospital. Due to the severe shortage of trained midwives, in 2007, the Obstetrical and Gynecological Society of Bangladesh (OGSB) proposed a short-term solution for training the existing nurse-midwives with a 6-month training program.

To provide quality midwifery services, three-year training after 12 years of general education scheme is developed. In 2010, a professional association, Bangladesh Midwifery Society (BMS) was founded as an advisory board to enhance the quality of Midwifery in Bangladesh. BMS also functions as a body to liaise with legislative and regulatory bodies as well as improve cooperation with national and international organizations. In 2013, the Community-Based Midwifery Diploma Programme (CMDP) was launched to provide a university qualification that would be compatible with national education. The students would be selected from areas where the maternal healthcare is needed the most. It includes a comprehensive systems approach, blending a "hub and spoke" education model with the guidelines proposed by WHO [16]. The program was formed with the intention of developing curriculum, determining the faculty development scheme, student selection procedure and evaluation, and deployment of the students after finishing the training. To secure the bases for the education model, the availability of properly located and implemented training facilities is also included as the basic function. Academic places for the diploma in midwifery education need the approval of the MoHFW and often require accreditation of the Bangladesh Nursing and Midwifery Council (BNMC). Although no legislation exists, recognizing midwifery as an autonomous profession until now, nevertheless, there exist some regulations. For example, to get licensed as Midwives, one must complete at least twenty supervised births in their academic curriculum. Midwives are allowed to provide injectable contraceptives or intrauterine devices and permitted to practice six of EmOC basic signal functions. Midwives working in the hospitals generally conduct the uncomplicated normal deliveries, and if complications arise, they need to be dealt with by the professional doctors. Midwives were allowed to administer drugs only if a doctor prescribes that.

Two fundamental concerns arise inasmuch as the success of midwifery is concerned; one is about the quality of midwifery education and training, and the other is the cultural acceptability of a midwife in the community. Since, as per the International Confederation of Midwives (ICM), midwives have the potential to complete the confederation approved midwifery education program and need to be registered to practice midwifery and use the title "midwife," therefore, a skilled midwife can reduce the additional workload of doctors and nurses. Bangladesh government has launched thirty-one institutions for training in midwifery, and with an intention of recruiting five hundred each year the government formed three thousand posts for midwives to be placed in distinct Upazila and Union Health Family Welfare Centers [17]. Upazila formerly called thana is a geographic region function as subunits of districts used principally for the government official activities. The functionality of an Upazila can be understood to be analogous to that of a borough or county of Western countries. Until May 2016, Bangladesh has 490 Upazilas. UNFPA is enabling the government to four critical areas: (1) establishing the midwifery program, (2) regulating and licensing for midwives, (3) quality education and faculty development, and (4) demand creation in the community. Near about 1289 certified midwives are serving in the government health facilities [18]. These midwives have completed their six-month duration basic course on midwifery, a training program supported by the UNFPA. The training program focuses the midwifery-led maternal and neonatal health services to assure the EmOC services. Since 2013, a three-year direct-entry diploma course on midwifery has been initiated by the Bangladesh government as well. Currently, thirty-one nursing institutions are giving training to over two thousand students, and at the end of the current year, five hundred students are supposed to complete their graduation and will be deployed as "midwives" to Upazila Health Complexes (UHC) to give 24/7 midwifery-led maternal care. According to the National Strategic Directions for Midwifery, there are five thousand FWVs in Bangladesh who, with a six-month training on midwifery, have become able to conduct normal deliveries [19]. One-year training can make them eligible for all kinds of midwifery services. In this vein, some fourteen private organizations, such as BRAC and other NGOs, are also offering a three-year diploma course in midwifery. UNFPA and a private institution like BRAC, with their ample financial contributions, the dedicated donors such as Canada and Sweden, and the DFID help to make quality midwifery service a reality. However, the service of midwives at the health complexes that are located in rural areas faces hurdles due to its dilapidated condition. The maternal and labor department is the most ignored ward in many clinics.

It is in this backdrop that MoHFW urged organizations like the UNFPA and the ICM to steer the implementation of the Midwifery Services Framework (MSF) to assure that freshly trained midwives are to be prepared to serve in an enabling context. The government has formed three thousand jobs for midwives to meet the pledge of the United Nations to the "Every Woman Every Child" plan [8]. Because of a sharp political engagement, the midwifery curriculum has gained its momentum recently. Maternal Health Strategy suggests that the country should reach a target of 98 percent deliveries taking place at facilities by 2030 [20]. The midwives will play a vital role; hence training midwives and forming a suitable working condition for them are essential. To assure the quality of training on midwifery, proficient faculty, a globally recognized curriculum, teaching assistant, a conducive learning atmosphere, dormitories, and suitable preservice practicum are essential factors. Community knowledge and appreciation supporting to the competencies and capacity of 
the midwives can contribute to the development of maternal and neonatal care. Developing the association with women to encourage self-care and wellness of mothers, infants, and exhibiting respect for the social dignity and for women as individuals with complete human rights are important as well. In addition, supporting women so that their opinions and decisions are heard and other culturally sensitive issues, such as discussing the healthcare matters with the midwives, need to be put in place to overcome practices that are harmful for women and babies. A focus on health improvement and infection prevention that see pregnancies as a natural life experience requires being ensured. Besides, the appreciation and approval of the Parliament of the Midwifery Act and regulation to protect the civil rights and midwives are crucial.

The second concern, the acceptability of the midwives in the community, where they were to deploy depends on the service quality itself. It is believed that quality training will assure quality service and quality service will drive to acceptability. Systematic monitoring and capability building mechanism for the trainers need to be assured. To ensure the quality of midwifery training, an impact evaluation of the existing curriculum is yet to be established. It is anticipated that acceptability can be attained with quality. If a woman receives good services in any healthcare facility, she will feel convenient to be there over and over again, and that may lead the whole community to accept the presence of that service. Eventually, the acceptability of midwives in the community will be settled. Hence, whether the effective management of skills is happening or not needs to be guaranteed first. Further, community-based recruitment is a decent option. A midwife from a distinct community knows better about her own inhabitants. Therefore, recruiting and training them from their place of origin can serve as means of reducing any frictions between the patient and the midwife. Further, the Directorate of Nursing needs to be authorized as the Directorate General of Nursing and Midwives to ensure that midwives stay where they are provided to deliver the required services, whereas the directorate can look after the necessities and inadequacies of midwives to meet their goals. The concerned authority needs to give maternity kit to the midwives so that they can assist the essential reproductive health-related aids to a woman. With the help of this kit, any midwife can conduct a delivery. Coordination with other healthcare providers is also under an obligation to foster a midwife-led care environment where a midwife can serve independently in an area. The midwife has to be the first contact person for any family and especially for the pregnant mother at the time of any sorts of complication.

Sociocultural disposition, financial accessibility, and geographical availability are yet another prominent issue as long as the success of midwifery profession is concerned. In 2012, of an estimated 154.7 million of Bangladesh population, 43.2 million women were in their reproductive age. The total fertility rate was 2.2. Near about 72 percent of the people of this country live in rural areas. It is expected that the total population of the country would reach about 185.1 million by 2030 , and about 4.3 million women at that time may seek the midwifery services. Of that demand, 66 percent of these in rural settings are projected [9]. Currently, there are large disparities in maternal behaviors and issues among rural and urban and between various wealth quintiles. Four or more antenatal care visits were found among 46 percent women in urban areas, whereas only 26 percent of women in rural areas make those visits. In the poorest wealth quintile, about 35 percent of women had received antenatal care from a medically trained provider, whereas, in the wealthiest quintile, 88 percent women of wealthiest quintile receive that antenatal care. About 73 percent of the wealthiest women visited at delivery by any medically qualified provider contrasted to only 18 percent of the poorest. To reach the goal of improving skilled birth attendance by a half, a precise and systematic approach to the training that stresses balance and quality are apparently needed along with supportive work conditions with suitable infrastructure and administration at the community level. It is clear that larger numbers of skilled maternal health professionals are needed who are competent and comfortable with working in communities and ready to negotiate effective and appropriate referral to the EmOC.

Although clinically a woman can follow a regular lifestyle during her pregnancy period, in Bangladesh, a pregnant woman is allowed to have restricted mobility and needs to adopt an exceptional lifestyle. Overall socioeconomic advancement, expanding education and empowerment of women, and reducing the fertility rate are important drivers behind the declines in MMR. Three delays increase MMR: (a) delay in recognizing the urgency, (b) delay in transporting to the higher-level facility, and (c) delay in receiving care [21]. To avoid these delays, the midwife is to develop a good working relationship with the acknowledged health service providers. Studies need to be advanced to know why the mortality rate varies sharply from division to division; the National Survey points that Rajshahi division has the highest mortality rate of 290 per 100000 per year, whereas it is much below compared to that rate in the neighboring divisions [5]. The nation needs a fresh novel perspective to accelerate reducing the preventable maternal and the deaths of the newborns. Mothers having access to a midwife before, during and after their pregnancy and during the time of delivery, have conferred to lessen maternal and newborn deaths noticeably. While a woman is pregnant, sufficient nutrition and standard prenatal care, comprising medication for maternal infections, retains women their health and strengthens the likelihood of a fresh newborn. These also improve the growth of the child, thereby generating a quadruple return on the expense. A preponderance number of these deaths are preventable, solely by having a professionally trained midwife attending women and infants.

From an economic perspective, spending money on the development of midwifery as a profession is also creditable step for the overall progress. The Bill \& Melinda Gates Foundation states that every dollar contributing to family planning can spare governments up to six dollars that can be allocated to advance health, housing, water, sanitation, and other public services [19]. Near about 23 percent of deliveries are executed through caesarean section in the country right now [22]. The rate is very startling and can be lessened by increasing in the number of midwives. A value for money evaluation conducted in 2013 reports 
that investing in midwives could generate a 16.2 return on investment [23]. Utilizing simply the number of caesarean sections (c-sections) avoided as the measure and considering that the rising trend for $\mathrm{c}$-sections in Bangladesh continues, community-based midwives could decrease the rate of increase significantly. For example, avoiding 3,391 c-sections per year or 101,719 over the next thirty years, at a net price of US $\$ 1,264 / \mathrm{c}$-section, the savings could reach about US\$128.5 million. Further, Institutionalization of Midwiferyled Infant and Maternity Care to depreciate the c-section rates can also reduce the pregnancy-related complications and even morbidity and strengthen the possibilities of child survival. In the situation as such, midwifery appears to be an affordable solution. The fund is another critical issue. Bangladesh spends only \$27 per person for health [23]. Health sector gets only 4.5 percent of the entire budget of the country, and in terms of GDP, it is merely one percent, whereas the health sector of the most developed nations constitutes nine percent of the total GDP [24]. Bangladesh needs to manage more funds with the belief that spending on midwives is an investment for the future. It is to say that a shift is needed from "a physician-led care to a midwifery-led maternal care."

Bangladesh Demographic Health Survey does not seem to be given proper attention to the stillbirths. Perinatal period extends from 28 weeks of gestation to first 7 days of life after birth [25]. Every death of a newborn in this period is designated as a perinatal death. Here, perinatal death audit plays a significant role in identifying avoidable factors to reduce the number by choosing appropriate responses against recognized preventable factors. Family planning is an alternative to decrease these unnecessary deaths. Empowering and educating women, which are fundamental for a society to ensure women's rights, can help families decide the frequency, timing, and spacing of pregnancies, turning into improved birth results for both the mothers and the babies. Further, the rigorous program needs to be adopted for ensuring good health and nutrition of the pregnant women, improvement of adolescent health, supervision of maternal diseases along with hypertension and diabetes, active monitoring of fetal growth, high-quality support at the time of delivery, prenatal audit, and high-quality antenatal care.

\section{Conclusion}

Midwives are the saviors where the stakes are lives of mothers and babies. They can foster confidence in the arrangement of birth giving. Globally, it is believed that childbirth is natural and wholesome and a woman is entitled to give birth without any form of nonevidence based medical invasion. A registered midwife can prevent this overmedicalization of the crude birth-giving process, and if there are no complexities, the midwife can encourage the community members to prefer routine delivery. Creating an environment conducive to midwives, the availability of all the necessary equipment needs to be ensured. Another important issue is tantamount to be certain of the quality of midwifery education curriculum. Recruiting experts on midwifery as teachers and trainers for the curriculum as such can guarantee the efficiency of the midwives. More midwifery education centers, more NGOs involvement, and better coordination among numerous health service providers of midwifery training and hiring should be the focal point to meet the demand for midwives. A partnership between the public and private sector, such as the midwives trained and educated in the private sector, and the infrastructure facilities provided by the public sector will benefit the reach of midwifery services to underprivileged communities. Community institutions can be useful in this regard as well. Further, both the government and private sector require working collectively and preparing a sufficient number of midwives in the coming days to overcome the situation. Creating an adequate number of faculties for midwifery education and deployment of the registered midwives immediately after the completion of their graduation are expected to be assured.

Bangladesh has no national level policy for midwives to guide their recruitment, appointment, and crucial issues as such. Now, they are chiefly member of the nursing pool. Hence, it is considerably anticipated that there will be tension between the midwives the nurses. A clear definition of midwifery can separate them out and dedicate them to reproductive health areas so that the maternal care can be strengthened. In addition, a suitable term for "midwife" (as the Bangla term "Dai" is not respectful, and an English word is not properly understood) should be properly acknowledged and accepted by everywhere to give the due respect to the profession. Midwives can do more than only catch the babies. The people of the country should give the profession proper respect. The support from the administrators of health facilities can help the midwives to perform their duty properly. The mass media, health service providers, policy-formulating groups, and the donors need to play a critical role to create awareness among people concerning the differentiation of these two professions. In addition, midwives can play a critical role in preventing early marriage and advancing women empowerment concerns. The midwifery educational institutions that are privately owned are better equipped than that of the public ones that often lack adequate faculties, hands-on training amenities, residences for students, and so on. Accreditation regularity for the public sector educational institutions to maintain quality is a crying need. That is why there should be a collaboration between public and private initiatives, and the government needs to spend more in midwifery training. It is necessary as midwifery is not only about skills but also about thinking like a mother and takes the right decision at the crucial moment with a certain level of conviction. Hence, licensing exam for registering the midwives should include more hands-on skills rather than only theoretical.

An untrained midwife should not carry out the deliveries as the safety of each and every single mother is of prime importance. Midwives in the United Kingdom render all the essential support to mothers and newborns, and solely the case of c-section patients is referred to the doctors. The educated midwives can provide similar forms of support in Bangladesh. Bangladesh government can make it incumbent for private health service providers to employ midwives in the maternity services. The principal thrust of the current 
midwifery program of Bangladesh is to send registered midwives to the community, where they are required the most. In addition, there are programs to introduce this novel professional to the societies, and there are plans to introduce Bachelor degree in midwifery in various educational institutions so that the midwifery education can be taken up to a higher stage. One noteworthy benefit would be the enhancement of skills of midwifery at the national level. Further, due to this degree, it can be expected that a pool of certified trainers who have bachelor degrees would be available to train those who are interested in becoming midwives in the coming years. In addition, it is an advancement to have a separate cadre in the public service commission of Bangladesh; consequently, a person with a BSS degree as such can make commendable contribute to shed lights on what would be required from the government in the future.

One of the major problems is that there is hardly any clear estimate of the number of pregnant women in Bangladesh, and without it, none can predict the demand of midwives. One estimate indicates that Bangladesh needs more than twenty thousand midwives, while only a few are registered. There ought to be a decent data administration method in this regard. One of the core SDG goals for Bangladesh is to decrease the MMR and to achieve it; there is no other alternatives to midwives. They can help in extending the coverage of family planning services. The Bangladesh government plans to deploy the newly graduated midwives, especially to the rural communities and improve the infrastructure for midwifery services. The government also has started a distant master's program on midwifery within distant learning format with the help of UNFPA. Finally, safe motherhood is a matter of any woman's right to choose from safe alternatives and right to survive. Registered midwives are under an obligation to be certain of these rights. Although pregnant women generally go to the obstetrician, in the developing nations, the system that ensures midwife will be the first contact person needs to be created. It requires making an appreciation of midwives in the society, where the people can feel that a trained midwife can render all the essential support for spontaneous delivery. There is an intolerable accelerating cost of deliveries these days. The midwives are more accessible than that of the doctors. That perhaps is cost effective as well. Creating demand for midwives will enhance the acceptability of them to the community and may ensure an enabling environment for midwives as well. Establishing a directorate of midwifery or forming a midwifery society comparable to nursing society can be useful.

\section{Conflicts of Interest}

The author declares that he has no conflicts of interest, neither financial nor nonfinancial and neither professional nor personal, regarding this paper.

\section{References}

[1] K. Taylor, Delivers: Quality Care for Expectant Moms Results in Fewer Stillbirths, USAID, 2016, https://blog.usaid.gov/2016/01/ usaid-delivers-quality-care-for-expectant-moms-results-infewer-stillbirths/.
[2] P. Mannava, K. Durrant, J. Fisher, M. Chersich, and S. Luchters, "Attitudes and behaviours of maternal health care providers in interactions with clients: a systematic review," Globalization and Health, vol. 11, no. 1, article 36, 2015.

[3] E. Fottrell, D. Osrin, G. Alcock et al., "Cause-specific neonatal mortality: analysis of 3772 neonatal deaths in Nepal, Bangladesh, Malawi and India," Archives of Disease in Childhood-Fetal and Neonatal Edition, 2015.

[4] J. E. Lawn, H. Blencowe, P. Waiswa et al., "Stillbirths: rates, risk factors, and acceleration towards 2030," The Lancet, vol. 387, no. 10018, pp. 587-603, 2016.

[5] J. Godinho, A. Vinokur, H. Tadesse, and T. Novotny, "Bangladesh-Poverty in Bangladesh: Building on Progress".

[6] J. H. Requejo, J. Bryce, and C. G. Victora, Building a Future for Women and Children: The 2012 Report, World Health Organization, 2012.

[7] A. Hossain, H. Moseson, S. Raifman, C. Gerdts, K. K. Biswas, and D. G. Foster, "'How shall we survive': a qualitative study of women's experiences following denial of menstrual regulation (MR) services in Bangladesh," Reproductive Health, vol. 13, no. 1, article 86, 2016.

[8] F. Gugsa, E. Karmarkar, A. Cheyne, and G. Yamey, "Newspaper coverage of maternal health in Bangladesh, Rwanda and South Africa: a quantitative and qualitative content analysis," $B M J$ Open, vol. 6, no. 1, Article ID e008837, 2016.

[9] UNFPA, WHO: The State of the World's Midwifery 2014: A Universal Pathway. A Women's Right to Health, United Nations Population Fund, New York, NY, USA, 2014.

[10] FIGO and ICM, Maternity Care in the World, 2nd edition, 1969.

[11] “Country MNCH Analysis Report for H4," Shorter Report 18 December 2009.

[12] V. Fauveau, K. Stewart, S. A. Khan, and J. Chakraborty, "Effect on mortality of community-based maternity-care programme in rural Bangladesh," The Lancet, vol. 338, no. 8776, pp. 11831186, 1991.

[13] 38-Country MNCH analysis shorter report, p. 16.

[14] S. N. Mitra, A. Al-Sabir, T. Saha, and S. Kumar, "Bangladesh Demographic and Health Survey 1999-2000," 2001.

[15] SoWMy questionnaire responded by Bangladesh in December 2010, February 2011.

[16] WHO, Global Recommendations for the Retention of Health Workers, World Health Organization, Geneva, Switzerland, 2010.

[17] M. U. Bogren, H. Wigert, L. Edgren, and M. Berg, “Towards a midwifery profession in Bangladesh-a systems approach for a complex world," BMC Pregnancy and Childbirth, vol. 15, no. 1, article 325, 2015.

[18] A. H. Chowdhury, K. M. Zahiduzzaman, and I. A. Bhuiya, “018: prospects, challenges, and way forward regarding deployment of midwives and professionalization of midwifery: evidence from developing midwives project in Bangladesh," BMJ Open, vol. 5, supplement 1, 2015.

[19] WHO, Report of the Sixth Meeting of the International Coordinating Group of the World Health Organization and the Bill \& Melinda Gates Foundation Project on Eliminating Human and Dog Rabies, Durban, South Africa, 22-24 September 2014, 2015.

[20] D. Godha, A. J. Gage, D. R. Hotchkiss, and C. Cappa, "Predicting maternal health care use by age at marriage in multiple countries," Journal of Adolescent Health, vol. 58, no. 5, pp. 504-511, 2016. 
[21] S. Thaddeus and D. Maine, "Too far to walk: maternal mortality in context," Social Science and Medicine, vol. 38, no. 8, pp. 10911110, 1994.

[22] I. Anwar, H. Y. Nababan, S. Mostari, A. Rahman, and J. A. M. Khan, "Trends and inequities in use of maternal health care services in Bangladesh, 1991-2011," PLoS ONE, vol. 10, no. 3, Article ID e0120309, 2015.

[23] A. Bhuiya and N. Chowdhury, "Determination of loss factors in a deregulated system," in Proceedings of the Canadian Conference on Electrical and Computer Engineering, Saskatoon, Canada, May 2005.

[24] M. A. Haque, S. K. Dash, and M. A. B. Chowdhury, "Maternal health care seeking behavior: the case of Haor (wetland) in Bangladesh," BMC Public Health, vol. 16, no. 1, article 592, 2016.

[25] D. S. Manandhar, "Perinatal death audit," Kathmandu University Medical Journal, vol. 2, no. 4, pp. 375-383, 2004. 


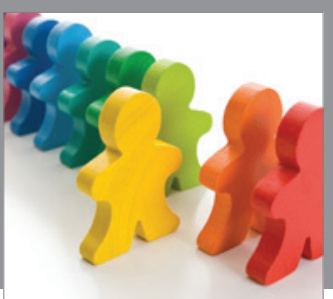

Autism

Research and Treatment
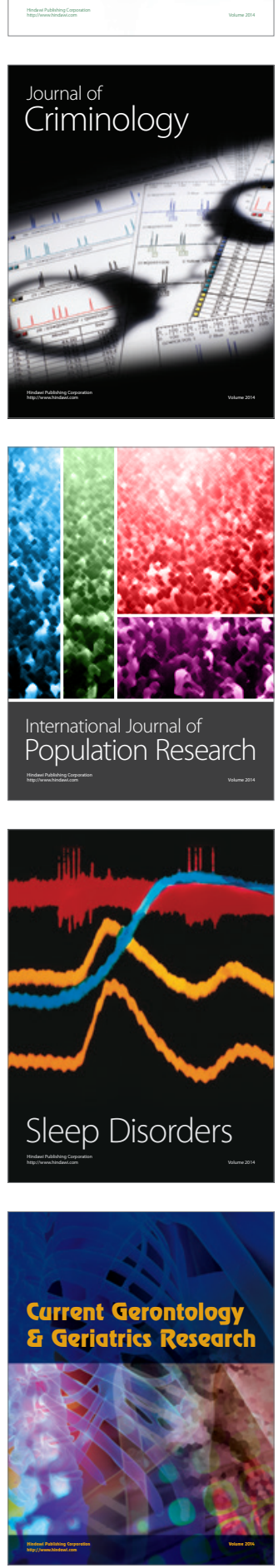

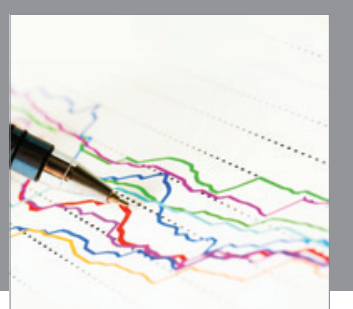

Economics

Research International
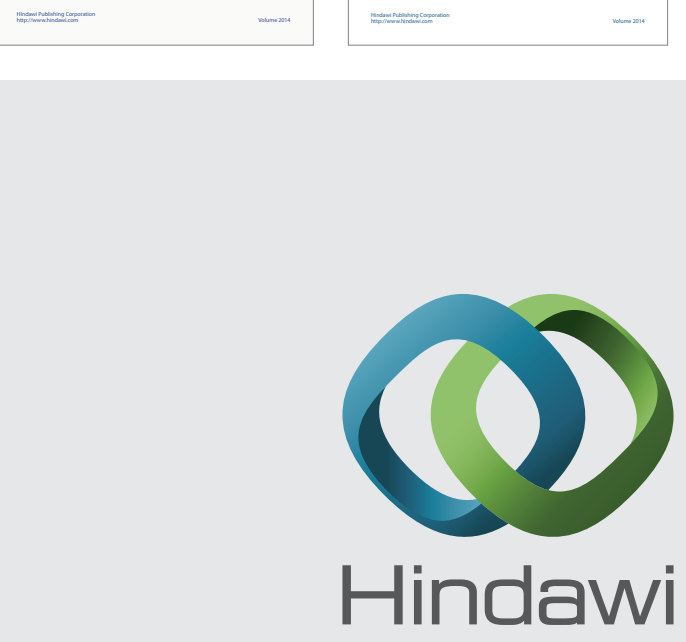

Submit your manuscripts at

https://www.hindawi.com
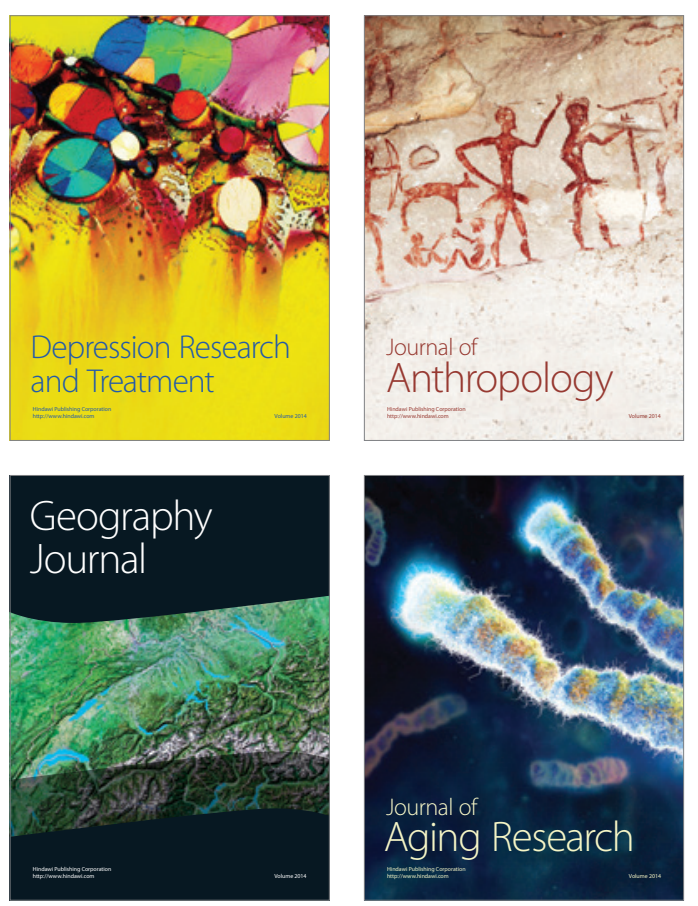
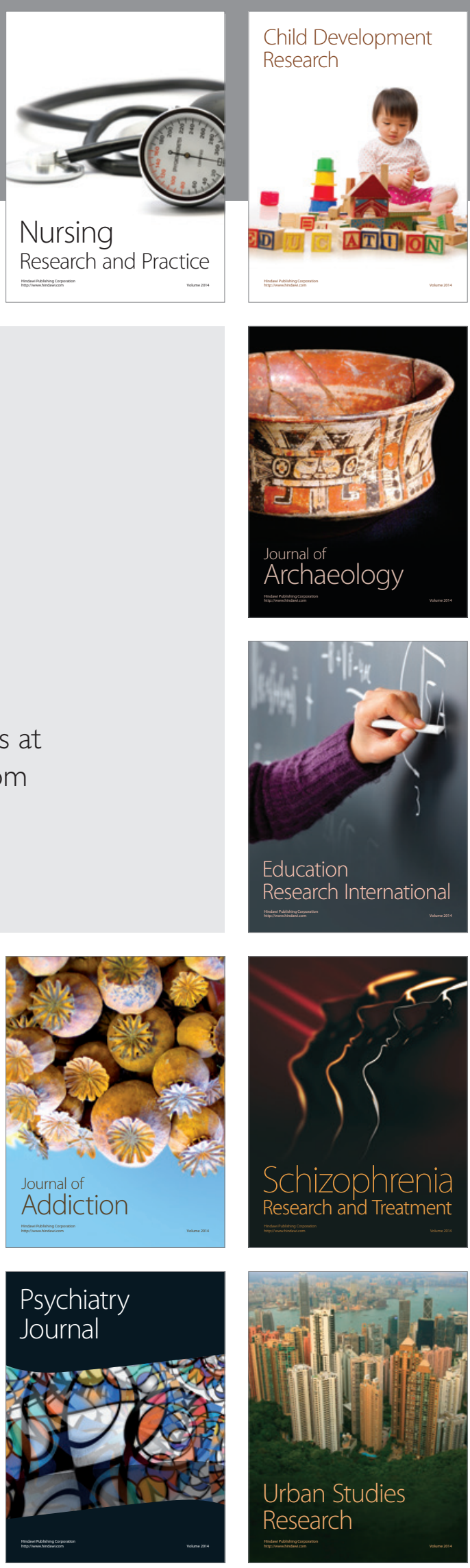